\title{
Local-structure change rendered by electronic localization-delocalization transition in cerium-based metallic glasses
}

\author{
Qiang Luo, ${ }^{1}$ Björn Schwarz, ${ }^{2}$ Janine C. Swarbrick, ${ }^{3}$ Jozef Bednarčik, ${ }^{4}$ Yingcai Zhu, ${ }^{5}$ Meibo Tang, ${ }^{6}$ Lirong Zheng, ${ }^{5}$ \\ Ran $\mathrm{Li}^{7}{ }^{7}$ Jun Shen, ${ }^{1, *}$ and Jürgen Eckert ${ }^{8,9}$ \\ ${ }^{1}$ School of Materials Science and Engineering, Tongji University, Shanghai 201804, People's Republic of China \\ ${ }^{2}$ Karlsruhe Institute of Technology (KIT), Institute for Applied Materials (IAM), 76344 Eggenstein-Leopoldshafen, Germany \\ ${ }^{3}$ European Synchrotron Radiation Facility (ESRF), BP22, 6 rue Jules Horowitz, 38043 Grenoble, France \\ ${ }^{4}$ Deutsches Elektronen Synchrotron DESY, Notkestrasse 85, D-22607 Hamburg, Germany \\ ${ }^{5}$ Institute of High Energy Physics, Chinese Academy of Sciences, Beijing 100049, China \\ ${ }^{6}$ Key Laboratory of Transparent and Opto-functional Inorganic Materials, Shanghai Institute of Ceramics, Chinese Academy of Sciences, \\ Shanghai 200050, People's Republic of China \\ ${ }^{7}$ Key Laboratory of Aerospace Materials and Performance (Ministry of Education), School of Materials Science and Engineering, Beihang \\ University, Beijing 100191, People's Republic of China \\ ${ }^{8}$ Erich Schmid Institute of Materials Science, Austrian Academy of Sciences, Jahnstraße 12, A-8700 Leoben, Austria \\ ${ }^{9}$ Department Materials Physics, Montanuniversität Leoben, Jahnstraße 12, A-8700 Leoben, Austria
}

(Received 30 August 2017; revised manuscript received 28 January 2018; published 12 February 2018)

\begin{abstract}
With increasing temperature, metallic glasses (MGs) undergo first glass transition without pronounced structural change and then crystallization with distinct variation in structure and properties. The present study shows a structural change of short-range order induced by an electron-delocalization transition, along with an unusual large-volume shrinkage in Ce-based MGs. An $f$-electron localization-delocalization transition with thermal hysteresis is observed from the temperature dependence of $\mathrm{x}$-ray absorption spectroscopy and resonant inelastic $\mathrm{x}$-ray scattering spectra, indicating an inheritance of the $4 f$ configuration of pure Ce. However, the delocalization transition becomes broadened due to the local structural heterogeneity and related fluctuation of $4 f$ levels in the Ce-based MGs. The amorphous structure regulated $4 f$ delocalization of Ce leads to bond shortening and abnormal structure change of the topological and chemical short-range orders. Due to the hierarchical bonding nature, the structure should change in a similar manner on different length scales (but not isostructurally like the Ce metal) in Ce-based MGs.
\end{abstract}

DOI: 10.1103/PhysRevB.97.064104

\section{INTRODUCTION}

The metallic bonding nature endows metallic glasses (MGs) with densely packed structure and unique local atomic configuration [1], which leads to many superior mechanical, magnetic, and chemical properties. The solute-centered atomic clusters are considered as the basic building blocks of MGs, but their packing schemes to form medium-range-order (MRO) structures remain elusive [2-6]. The local structure change of MG with composition has been intensively investigated, focusing on glass formation and material design with required properties $[1,4]$. Besides, temperature and pressure have been used to tune the short-range order (SRO), MRO, and properties of MGs [7-11]. The investigation of structure change with temperature is mainly focused on glass transition and crystallization events in MGs [1,7,11]. Recently, pressureinduced amorphous-to-amorphous transition has been reported in some rare-earth-based MGs, which originates from the change of $4 f$ electronic configuration $[12,13]$, which attracts intensive interest. These works indicate a significant impact of electronic configuration on the structure of MGs and offer a new perspective of the amorphous configuration of MGs.

\footnotetext{
*Corresponding author: junshen@tongji.edu.cn
}

Actually, since the work of Nagel and Tauc, it has been realized that the electronic configuration near the Fermi level correlates with the glass-forming ability of MGs [14,15]. Moreover it was found that the strength and ductility of MGs depended on the detailed features of $s$-character/pd-hybrid bonds $[16,17]$. The correlation between atomic structure and electronic configuration underpins our understanding of the nature of MGs, which is still a longstanding challenge.

Here we report, at ambient pressure and below room temperature, a local-structure change of topological and chemical short-range orders, along with an unusual large-volume shrinkage in two Ce-based MGs. The Ce L3-edge x-ray absorption spectroscopy (XAS) measurements, along with results of the $\mathrm{Ce} 2 p 3 d$ resonant inelastic x-ray scattering (RIXS) spectra showing well-resolved features of the unoccupied $\left(f^{0}\right)$ and singly $\left(f^{1}\right)$ and doubly $\left(f^{2}\right)$ occupied $4 f$ states, uncover a $4 f$-electron localization-to-delocalization transition as the main mechanism of the abnormal local-structure change and unusual large-volume contraction.

\section{EXPERIMENTAL DETAILS}

Rod-shaped $\mathrm{Ce}_{62} \mathrm{Al}_{10} \mathrm{Cu}_{20} \mathrm{Co}_{8} \mathrm{MG}$ with a diameter of $3 \mathrm{~mm}$ was prepared using the suction-casting method. The 

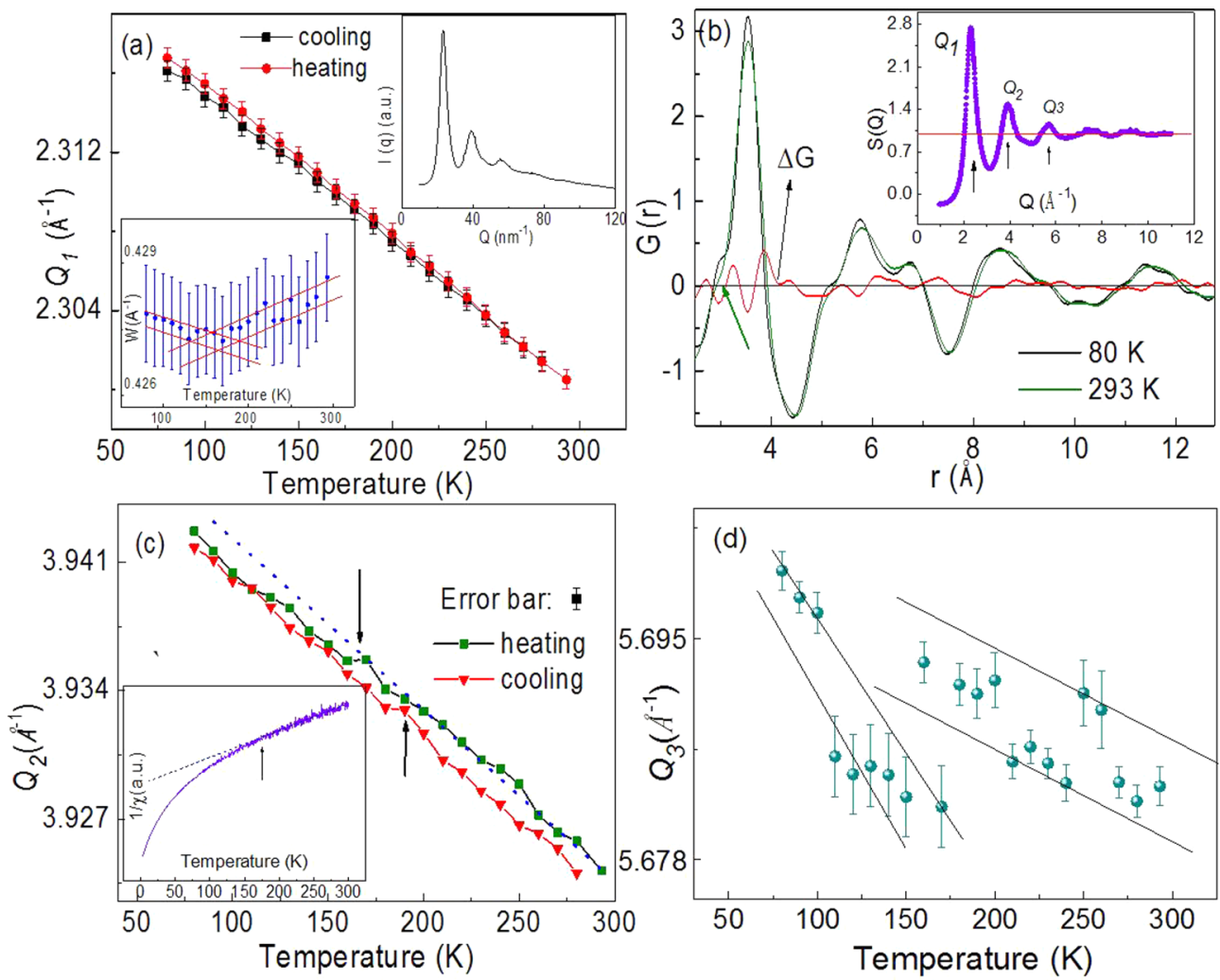

FIG. 1. (a) The change of FSDP of synchrotron XRD. The upper inset shows the XRD at $293 \mathrm{~K}$ for $\mathrm{Ce}_{65} \mathrm{Al}_{10} \mathrm{Co}_{25}$. The lower inset shows the change of the full width at half maximum of the FSDP during heating. (b) Pair-distribution functions, $G(r)$, of $\mathrm{Ce}_{65} \mathrm{Al}_{10} \mathrm{Co}_{25}$ at 80 and $293 \mathrm{~K}$. The red line is their difference. The inset shows the $S(Q)$ at $293 \mathrm{~K}$. (c) Change of the second peak of $S(Q)$; the inset shows the inverse susceptibility of $\mathrm{Ce}_{65} \mathrm{Al}_{10} \mathrm{Co}_{25}$. (d) Evolution of the third peak of $S(Q)$ during heating.

$\mathrm{Ce}_{65} \mathrm{Al}_{10} \mathrm{Co}_{25}$ glassy ribbon was prepared using the singleroller melt-spinning technique. In situ low-temperature Ce L3edge XAS experiments were performed at the 1W1B-XAFS beamline of the Beijing Synchrotron Radiation Facilities in Beijing, China. Transmission mode was employed, and the samples were polished down to approximately $10 \mu \mathrm{m}$ in thickness. The RIXS experiments were performed at the ID26 beamline of the European Synchrotron Radiation Facilities. The incident energy at different emission energies around the $L_{\alpha 1}$ emission line was scanned to measure the full RIXS plane. The X-ray appearance near-edge structures (XANES) were simultaneously measured in total fluorescence yield (TYF) mode using a photodiode and in high-energy-resolution fluorescence-detected (HERFD) mode with an X-ray emission spectrometer. A liquid He cryostat was used to maintain the cryogenic temperatures of the sample during the lowtemperature RIXS measurements.

In situ low-temperature X-ray diffraction (XRD) experiments using high-energy photons were performed at the P02.1 undulator beamline of the PETRA III electron storage ring at DESY (Hamburg, Germany). XRD patterns were acquired under transmission mode. The energy of the synchrotron radiation was set to $59.84 \mathrm{keV}$, which corresponds to a wavelength of $\lambda=0.2072 \AA$. The temperature of the sample was adjusted by blowing a cold stream of gaseous $\mathrm{N}_{2}$ generated by the Oxford CryoStream 700 cryocooler. At each temperature, the sample was illuminated for $10 \mathrm{~s}$ by an incident beam with a cross section of $0.5 \mathrm{~mm} \times 0.5 \mathrm{~mm}$. Two-dimensional XRD patterns were collected using a Perkin Elmer 1621 Fast Image Plate Detector (2048 pixels $\times 2048$ pixels, $200 \mu \mathrm{m} \times 200 \mu \mathrm{m}$ pixel size, and an intensity resolution of 16 bit) carefully mounted orthogonal to the x-ray beam. The sample-to-detector distance was set to $498 \mathrm{~mm}$, implying that a $q$ range $[q=4 \pi \sin (\theta) / \lambda$, where $2 \theta$ is the scattering angle] of up to $14 \AA^{-1}$ was covered. The standard $\mathrm{CeO}_{2}$ powder material was used to calibrate the sample-to-detector distance and the degree of tilting of the imaging plate relative to the beam path. The $2 \mathrm{D}$ XRD patterns were integrated into the $q$ space using the FIT2D software package.

Thermal expansion $\Delta L / L(300 \mathrm{~K})$ was measured using a foil strain gauge (KYOWA; model KFL-02-120-C1). The gauge was bonded with PC-6 and measured using the Physical Property Measurement System (PPMS; Quantum Design Inc.). The corresponding thermal expansion data of pure Al (purity: 99.99\%) were used to calibrate the gauge. Magnetization was measured using the PPMS.

\section{RESULTS AND DISCUSSION}

First we performed temperature-dependent synchrotron XRD experiments on the $\mathrm{Ce}_{65} \mathrm{Al}_{10} \mathrm{Co}_{25} \mathrm{MG}$. A typical XRD pattern measured at $293 \mathrm{~K}$ is shown in the inset of Fig. 1(a), indicating the amorphous nature. As seen in Fig. 1(a), the first sharp diffraction peak (FSDP), $Q_{1}$, shifts almost linearly toward higher value with decreasing temperature. However, the full width at half maximum of the FSDP shows a crossover 
behavior around 164-169 K [the lower inset of Fig. 1(a)], indicating a significant local structural rearrangement. The distinct structure difference can further be revealed from the pair-distribution functions obtained at 80 and $293 \mathrm{~K}$ [Fig. 1(b)]. The first maximum and its shoulder locate around 3.54 and $3 \AA$, which is approximately in accordance with the $\mathrm{Ce}-\mathrm{Ce}$, and $\mathrm{Ce}-\mathrm{Al} / \mathrm{Co}$ nearest-neighbor distances, respectively. When decreasing the temperature from 293 to $80 \mathrm{~K}$, the first maximum of $G(r)$ reduces about $0.11 \%$. In addition, the shape of the first and second maxima is altered and their amplitude increases with decreasing temperature. Besides, the shoulders around 3 and 5.2 A develop obviously with decreasing temperature. These changes indicate a redistribution of the atoms within the first- and second-nearest-neighbor shells. On the other hand, from $G(r)$ the coordination numbers are determined to be 12.69 for $T=80 \mathrm{~K}$ and 12.77 for $T=300 \mathrm{~K}$, respectively. Therefore, the total nearest-neighbor number of the sample almost does not depend on temperature.

The local structural change can be indicated more clearly from the evolution of the second and third peak positions of structure factor, $S(Q)$, with temperature. Figure 1(c) shows a clear change of $Q_{2}$ centered around $\sim 170 \mathrm{~K}$ during heating, above which the data follow a different trajectory from that below. The most obvious structure change is revealed from the temperature dependence of the third peak of $S(Q), Q_{3}$. As indicated in Fig. 1(d), $Q_{3}$ shows a distinct sudden change around $170 \mathrm{~K}$ during heating. It is generally accepted that $Q_{1}$ reflects the MRO correlation in real space, while the high- $Q$ peaks $\left(Q_{2}, Q_{3}\right.$, etc.) embody more $\mathrm{SRO}$ features $[9,10]$.The different temperature dependence of $Q_{1}, Q_{2}$, and $Q_{3}$ indicates that local structure change occurs mainly on the SRO scale. To check whether this abnormal structure change is related to magnetic ordering/transition, we investigated the temperaturedependent magnetization. As seen from the inset of Fig. 1(c) showing the inverse magnetic susceptibility of $\mathrm{Ce}_{65} \mathrm{Al}_{10} \mathrm{Co}_{25}$, no magnetic transition or freezing is observed at least down to $2 \mathrm{~K}$ for the sample.

Since there is no glass transition or magnetic transition, the abnormal local structural change may arise from the variation of electron configuration. XAS and RIXS experiments were thus conducted to explore the change of $4 f$ electronic configurations. Figure 2(a) shows the Ce L3-edge XAS spectra obtained at different temperatures during heating. All of the spectra exhibit a $4 f^{1}$ component peak and a $4 f^{0}$ component bump, demonstrating a majority of localized with a few delocalized $4 f$ configurations. With increasing temperature, the bump slightly changes but the $4 f^{1}$ peak intensity increases obviously. Interestingly, the temperature dependence of the peak intensity shows a crossover behavior around 155-168 K [Fig. 2(b)], suggesting a significant change in the $4 f$ configurations. More details on the $4 f$ configuration change can be revealed from the $L$-edge RIXS experiments, which can probe the mixed-valence behavior of rare-earth compounds more efficiently than conventional XAS $[18,19]$. Figure 2(c) shows the HERFD XANES spectrum, along with the TFY curve recorded at $10 \mathrm{~K}$ for $\mathrm{Ce}_{62} \mathrm{Al}_{10} \mathrm{Cu}_{20} \mathrm{Co}_{8}$. The HERFD spectrum exhibits a better signal-to-noise ratio compared with that of the TFY curve and reveals better-resolved structures $\left(f^{2}\right.$ and $f^{0}$ features), benefited by the smaller broadening of the shallow $3 d$ hole than that of the deep $2 p$ core hole in the final
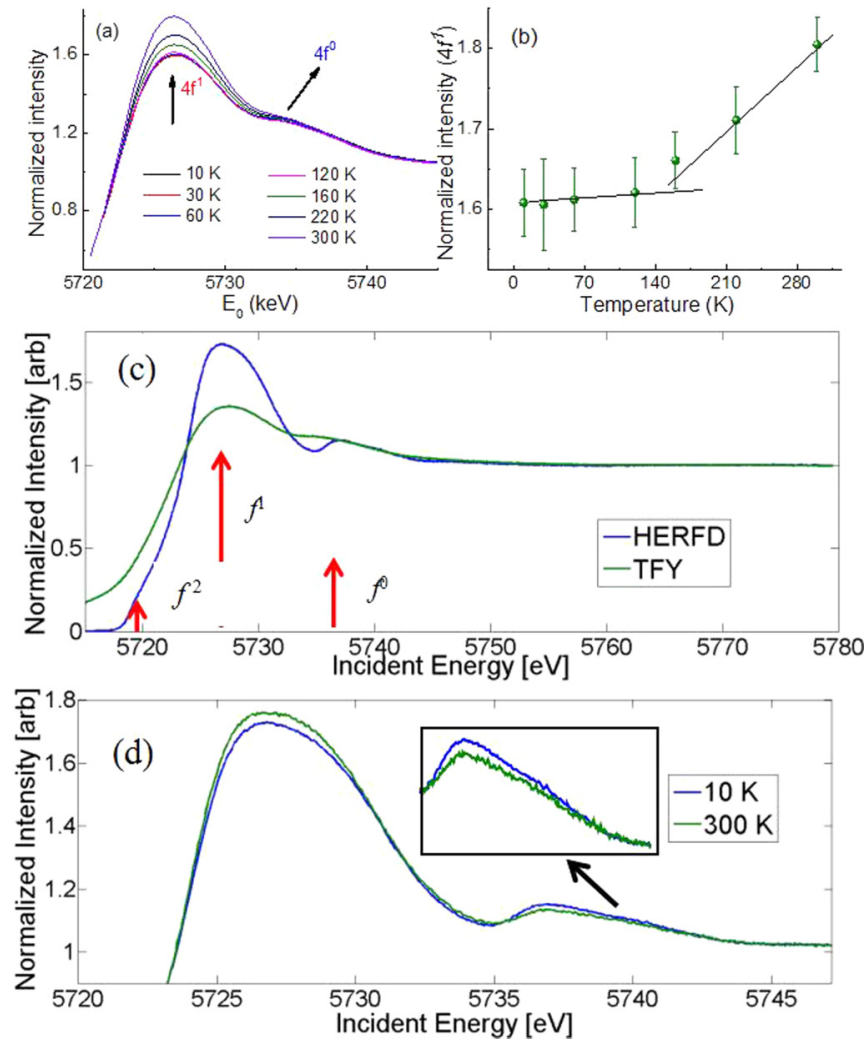

FIG. 2. (a) Ce $L 3$-edge XAS spectra of $\mathrm{Ce}_{65} \mathrm{Al}_{10} \mathrm{Co}_{25}$. The arrows point to the $4 f^{0}$ and $4 f^{1}$ components. (b) The change of the $4 f^{1}$ peak intensity with temperature. (c) HERFD Ce $L_{3}$ XANES spectrum of $\mathrm{Ce}_{62} \mathrm{Al}_{10} \mathrm{Cu}_{20} \mathrm{Co}_{8}$ recorded with the $\mathrm{x}$-ray emission energy set to the maximum of $L_{\alpha 1}$ at $10 \mathrm{~K}$. The spectrum is compared with the TFY curve recorded using a photodiode. (d) HERFD spectra recorded at 10 and $300 \mathrm{~K}$ for $\mathrm{Ce}_{65} \mathrm{Al}_{10} \mathrm{Co}_{25}$.

state [18]. The HERFD spectrum of the Ce-based MGs displays the following behavior: as the incident energy is tuned to the preedge region, a shoulder appears on the low energy-transfer side of the main emission. This feature resonates at $E_{0}$ of $\sim 5718.9 \mathrm{eV}$ [denoted as $f^{2}$ in Fig. 2(c)], which is not resolved in the TFY curve. The $f^{2}$ feature of the Ce metal was predicted by the Anderson impurity model but was not observed for a long time until the usage of the RIXS technique [19]. With increasing incident energy, the intensity continues to grow until it reaches a maximum at the white line energy, and the main peak at $\sim 5725.4 \mathrm{eV}$ (denoted as $f^{1}$ ) reflects the $3 d^{9} 4 f^{1} L 5 d$ character $(L$ is a hole in the ligand orbital). The high-energy bump [ $\sim 5736.4 \mathrm{eV}$, denoted as $f^{0}$ in Fig. 2(c)] is ascribed to the main $3 d^{9} 4 f^{0} 5 d$ component, which can be clearly observed in the HERFD curve but only appears as a shallow hump in the TFY curve.

The improved signal-to-noise ratio achieved through RIXS permits precise determination of the variation of $f$ configurations with temperature. Figure 2(d) shows the HERFD spectra of $\mathrm{Ce}_{65} \mathrm{Al}_{10} \mathrm{Co}_{25}$ recorded at 300 and $10 \mathrm{~K}$ comparatively. When going from 10 to $300 \mathrm{~K}$, the $f^{0}$ bump decreases, the $f^{1}$ peak increases [Fig. 2(d)], and the $f^{2}$ feature stays nearly unchanged. The transfer of spectral weight from $f^{1}$ to $f^{0} \mathrm{com}$ ponents clearly indicates the electron delocalization. Figure 3 shows that the $f^{1} / f^{0}$ ratio of both samples, as a qualitative 


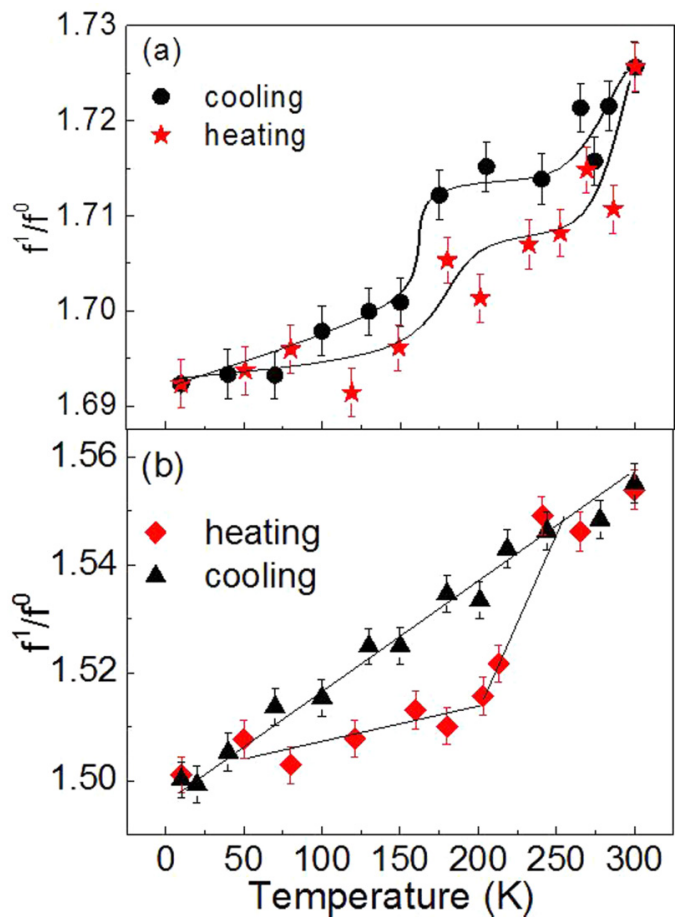

FIG. 3. Variation of the intensity ratio $f^{1} / f^{0}$ for $\mathrm{Ce}_{62} \mathrm{Al}_{10} \mathrm{Cu}_{20} \mathrm{Co}_{8}$ (a) and $\mathrm{Ce}_{65} \mathrm{Al}_{10} \mathrm{Co}_{25}$ (b).

indicator, decreases continuously with decreasing temperature. An obvious hysteretic behavior is observed between heating and cooling processes, pointing to a localization-delocalization transition. A closer comparison of the heating and cooling curves of $\mathrm{Ce}_{65} \mathrm{Al}_{10} \mathrm{Co}_{25}$ reveals that the transition may start at approximately $240 \mathrm{~K}$ where bifurcation occurs. The largest difference of the two curves occurs at approximately $170 \mathrm{~K}$, where a clear crossover takes place during heating and the distinct local structure change is observed [Fig. 1(d)]. Above $240 \mathrm{~K}$ or below $50 \mathrm{~K}$, the two $f^{1} / f^{0}$ curves agree with each other, suggesting the broadness and reversible nature of the transition.

To show more detailed information on the change of electronic configuration, the full RIXS plane around the $L_{\alpha 1}$ $\mathrm{X}$-ray emission line excited with photon energies across the $L_{3}$ edge for the Ce-based MGs at 10 and $300 \mathrm{~K}$ are shown as contour maps in Figs. 4(a)-4(d), in which the variations of the color reflect different intensities. A full RIXS plane for the sample scanned with incident energy at different emission energies around the $L_{\alpha 1}$ emission line was recorded in less than one hour. Such a scan in the maximum of the $L_{\alpha 1}$ emission line is referred to as HERFD absorption. The RIXS spectra as obtained in this study show the clear advantage over normal $L$-edge XANES studies in their ability to completely resolve the $f^{1}, f^{0}$, and $f^{2}$ features present, as well as revealing features in the RIXS plane. The different electronic configurations of the low- and high-temperature phases can be clearly revealed from the distinct details of the patterns shown in Fig. 4 (e.g., see the area in the incident energy range of 5735-5750 eV and the emitted energy range of $4838-4841 \mathrm{eV}$ for $\mathrm{Ce}_{65} \mathrm{Al}_{10} \mathrm{Co}_{25}$ ).

The above results indicate clearly the electronic mechanism of the abnormal local structural change. In crystalline $\mathrm{Ce}$,
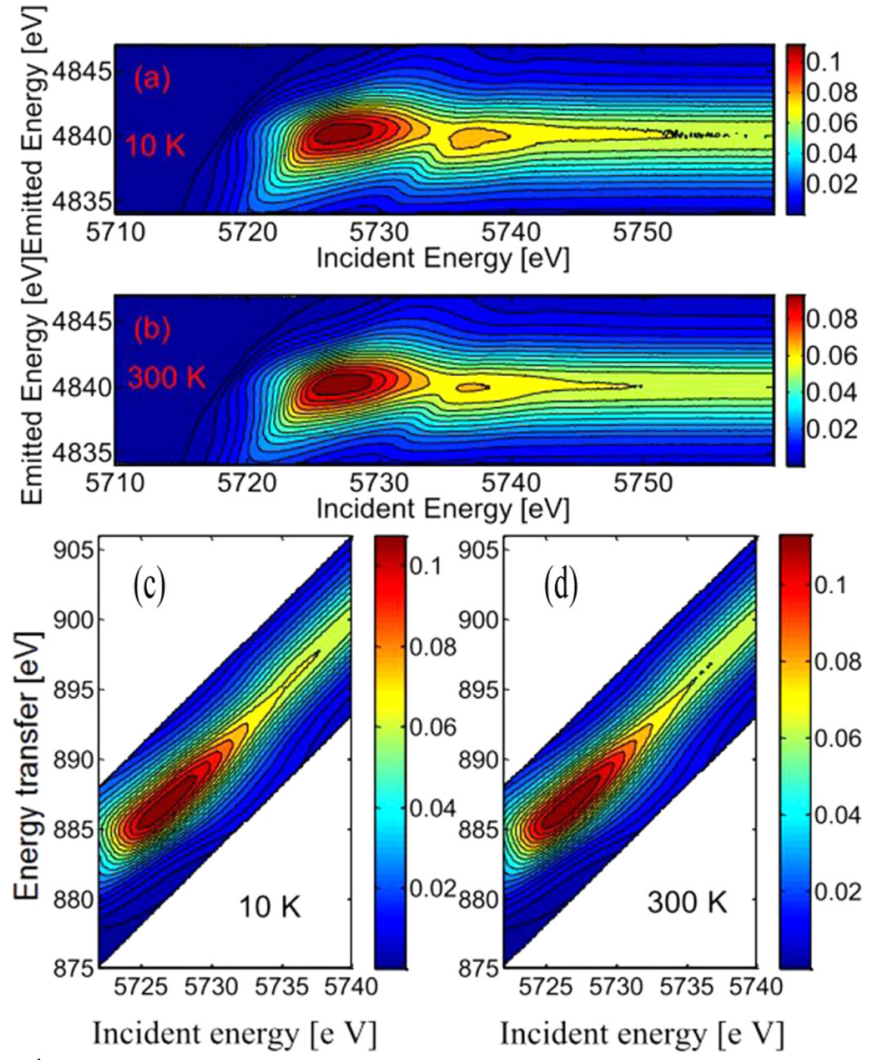

FIG. 4. Full RIXS intensities displayed as contour maps in planes for $\mathrm{Ce}_{65} \mathrm{Al}_{10} \mathrm{Co}_{25}$ at $300 \mathrm{~K}$ (a) and $10 \mathrm{~K}$ (b). Full RIXS intensities displayed as contour maps for $\mathrm{Ce}_{62} \mathrm{Al}_{10} \mathrm{Cu}_{20} \mathrm{Co}_{8}$ at $10 \mathrm{~K}$ (c) and $300 \mathrm{~K}(\mathrm{~d})$.

the $4 f$-electron configuration change induced a fascinating isomorphic transition with large volume collapse with applied pressure or reduced temperature. To explore the macroscopic volume shrinkage in Ce-based $\mathrm{MGs}$, we investigated the thermal expansion of $\mathrm{Ce}_{62} \mathrm{Al}_{10} \mathrm{Cu}_{20} \mathrm{Co}_{8}$ and several other bulk MGs from 5 to $300 \mathrm{~K}$. Figure 5(a) displays the relative length change $-\Delta L / L(300 \mathrm{~K})$ between 5 and $300 \mathrm{~K}$ for typical bulk MGs (one from other investigation [20]). The Ce-based MGs show the largest relative length change and thus the largest volume shrinkage. The relative length changes $[L(300 \mathrm{~K})-L(5 \mathrm{~K})] / L(300 \mathrm{~K})$ are approximately $0.47 \%$ (relative volume change, $\Delta V / V \sim 1.4 \%$ ) for $\mathrm{Ce}_{62} \mathrm{Al}_{10} \mathrm{Cu}_{20} \mathrm{Co}_{8}$, $0.22 \%(\Delta V / V \sim 0.6 \%)$ for $\mathrm{Zr}_{64.13} \mathrm{Cu}_{15.75} \mathrm{Ni}_{10.12} \mathrm{Al}_{10}$, and $0.17 \%$ $\left(\Delta V / V \sim 0.5 \%\right.$ ) for $\mathrm{Ho}_{30} \mathrm{Al}_{24} \mathrm{Co}_{20} \mathrm{Y}_{26}$. The lattice contraction of $\mathrm{Ce}_{62} \mathrm{Al}_{10} \mathrm{Cu}_{20} \mathrm{Co}_{8}$ can be twice as large as (even larger than) that of other bulk MGs, suggesting that approximately half of the volume contraction is due to $4 f$ electronic delocalization. This scenario is consistent with the large softening of the longitudinal acoustic mode and the large reduction of the bulk modulus with decreasing temperature reported in $\mathrm{Ce}_{68} \mathrm{Al}_{10} \mathrm{Cu}_{20} \mathrm{Co}_{2} \mathrm{MG}[21,22]$. Although the volume shrinkage of $\mathrm{Ce}_{62} \mathrm{Al}_{10} \mathrm{Cu}_{20} \mathrm{Co}_{8}$ is smaller than that of $\mathrm{Ce}$ metal, in fact the volume shrinkage of $\sim 1.4 \%$ is unusually large for MGs. Note that after crystalline transition the volume difference between a bulk $\mathrm{MG}$ and its fully crystallized counterpart is usually $0.3-1 \%$ [1]. For $\mathrm{Ce}_{62} \mathrm{Al}_{10} \mathrm{Cu}_{20} \mathrm{Co}_{8}$, we also found that the relative length change $[L(558 \mathrm{~K})-$ 


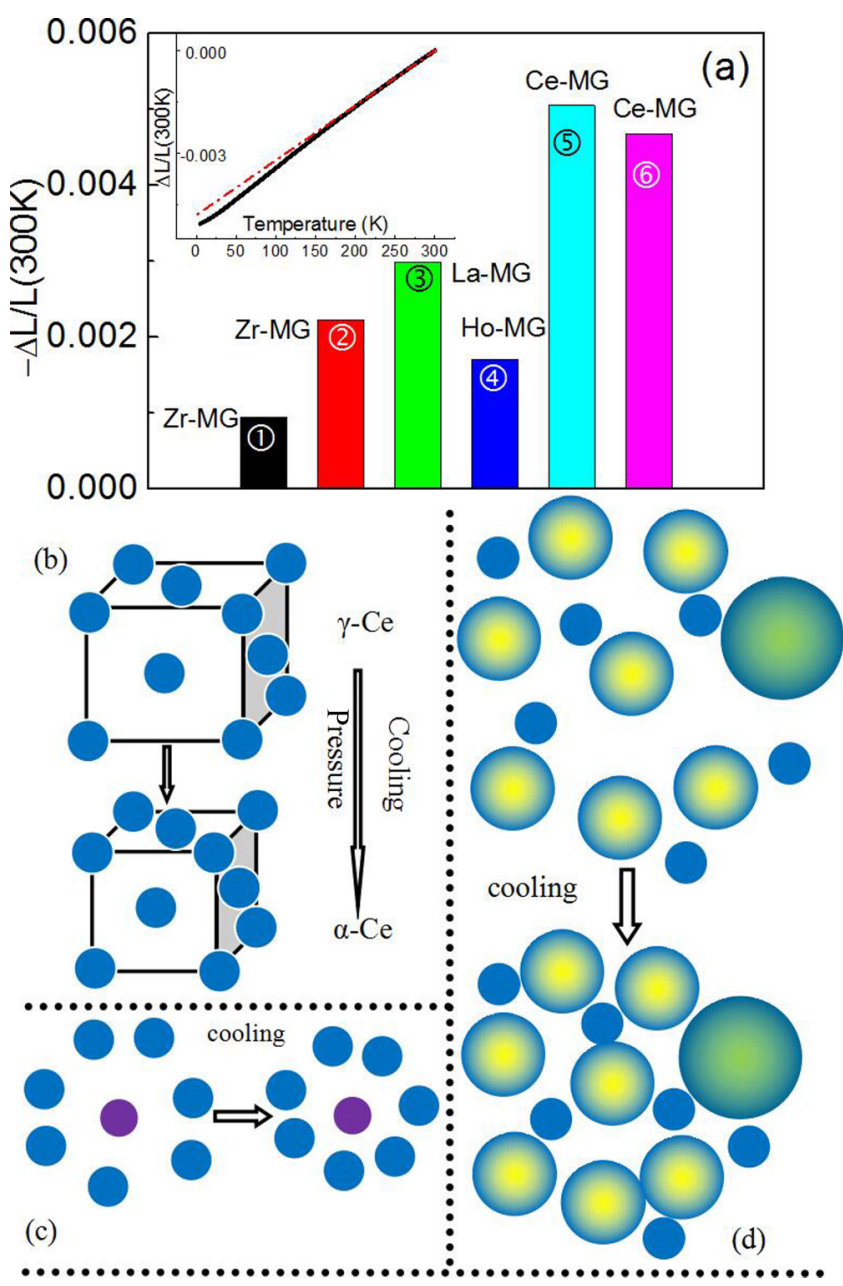

FIG. 5. (a) Relative length change of typical MGs: (1) $\mathrm{Zr}_{41} \mathrm{Ti}_{14} \mathrm{Cu}_{12.5} \mathrm{Ni}_{10} \mathrm{Be}_{22.5}$, (2) $\mathrm{Zr}_{64.13} \mathrm{Cu}_{15.75} \mathrm{Ni}_{10.12} \mathrm{Al}_{10}$, (3) $\mathrm{La}_{68} \mathrm{Al}_{10} \mathrm{Cu}_{20} \mathrm{Co}_{2}$, (4) $\mathrm{Ho}_{30} \mathrm{Al}_{24} \mathrm{Co}_{20} \mathrm{Y}_{26}$, (5) $\mathrm{Ce}_{68} \mathrm{Al}_{10} \mathrm{Cu}_{20} \mathrm{Co}_{2}$, (6) $\mathrm{Ce}_{62} \mathrm{Al}_{10} \mathrm{Cu}_{20} \mathrm{Co}_{8}$. The inset shows the relative length change of $\mathrm{Ce}_{62} \mathrm{Al}_{10} \mathrm{Cu}_{20} \mathrm{Co}_{8}$ with increasing temperature. (b) Isostructural $\gamma-\alpha$ transition of metal Ce. (c) Illustration of the change of a SCC with cooling (the blue ball represents the $\mathrm{Ce}$ atom, and the pink ball the solute atom $\mathrm{Al}$ or $\mathrm{Co}$ ). (d) Illustration of the change of an extended structure (beyond the nearest-neighbor shell) packing of weakly bonded solvent Ce atoms, SCCs (medium-size balls), and superclusters (the biggest ball).

$L(300 \mathrm{~K})] / L(300 \mathrm{~K})$ is approximately $0.46 \%$ upon heating up to $558 \mathrm{~K}$ (above the crystallization temperature $\sim 458 \mathrm{~K}$ ). This indicates a similar volume change for the localizationdelocalization transition and the glass-to-crystalline phase transition in $\mathrm{Ce}_{62} \mathrm{Al}_{10} \mathrm{Cu}_{20} \mathrm{Co}_{8}$. In addition, temperature dependence of $\Delta L / L$ for $\mathrm{Ce}_{62} \mathrm{Al}_{10} \mathrm{Cu}_{20} \mathrm{Co}_{8}$ deviates from a linear behavior from around $170 \mathrm{~K}$ [the inset of Fig. 5(a)], where the abnormal local structure change occurs.

In a unique manner among all elements, Ce undergoes an isostructural $\gamma$-to- $\alpha$ transition [Fig.5(b)] [23,24], in which the $4 f$-electron localization and screening effect plays a crucial role (although the detailed mechanism remains debatable [23-28]). In contrast to the periodic arrangement of atoms in $\mathrm{Ce}$ metal, $\mathrm{Ce}$ ions are strongly disordered in Ce-based MGs. On the other hand, MGs exhibit SRO, characterized by solute-centered clusters (SCCs), and MRO formed by packing of SCCs via various proposed schemes [2-6]. Nevertheless, our results indicate that the disordered $\mathrm{Ce}$ atoms in $\mathrm{MGs}$ inherit largely the $4 f$ configuration features, especially the delocalization transition, of elemental Ce. The $f$-electron delocalization can promote the low-lying $f$ electrons close to the Fermi level, resulting in more $4 f$ electrons participating in electronic bonding and metallic bond shortening. Thus, the unusually large volume shrinkage in the Ce-based MGs arises from $f$-electron delocalization process as in the Ce metal. In contrast to the isostructural transition in elemental $\mathrm{Ce}$, the changes in SRO and MRO of Ce-based MGs promoted by $f$-electron delocalization are diverse depending on their local environments.

Figures 5(c) and 5(d) illustrate schematically the local structural change in $\mathrm{Ce}_{65} \mathrm{Al}_{10} \mathrm{Co}_{25}$ upon cooling. Based on the dense cluster packing models and the concept of topological fluctuations and local atomic stresses of MGs [2-5,29,30], the structure of $\mathrm{Ce}_{65} \mathrm{Al}_{10} \mathrm{Co}_{25} \mathrm{MG}$ is considered as a packing of tightly bonded SCCs (including a few superclusters, each of which is made up of a solute $\mathrm{Al}$ or Co atom surrounded by mostly solvent Ce atoms). In addition, some loosely packed glue/excess atoms (rich in $\mathrm{Ce}$ ) form the liquidlike regions. We mainly focus the $\mathrm{Ce}-\mathrm{Ce}$ bonds because they dominate the structural change. The amorphous nature of $\mathrm{Ce}_{65} \mathrm{Al}_{10} \mathrm{Co}_{25}$ means a hierarchical bonding characteristic of the $\mathrm{Ce}-\mathrm{Ce}$ bonds. The neighboring $\mathrm{Ce}-\mathrm{Ce}$ bonds within a SCC process the strongest bonding forth, whereas the Ce-rich liquidlike regions demonstrate the weakest $\mathrm{Ce}-\mathrm{Ce}$ bonding. Moreover, the intercluster $\mathrm{Ce}-\mathrm{Ce}$ bonds, which connect nearest-neighbor SCCs to form icosohedral-like and/or crystal-like order, have medium-strength interaction. Fluctuations in the $\mathrm{Ce}-\mathrm{Ce}$ bonding strength in the Ce-based MGs induce related fluctuations of the local density of states, and distribution of mixing between $4 f$ and conduction electrons [31,32]. Structurally, upon cooling a SCC would shrink accompanied by shortening of the surrounding Ce-Ce bonds as illustrated in Fig. 5(c), which is enhanced by the delocalization transition. During shrinkage, the SCC can be distorted because the outer Ce atoms of the SCC "experience" different local stresses. This SCC's shrinkage and rearrangement gives rise to the transition of the chemical and topological short-range orders. An increase in the number of $\mathrm{Ce}-\mathrm{Ce}$ nearest-neighbor pairs thus may be possible during the chemical short-range ordering because of the strong $4 f$-electron correlation. This is similar to the chemical shortrange-ordering process during annealing, which is associated with an increase of the number of $\mathrm{Fe}-\mathrm{Fe}$ nearest-neighbor pairs in an Fe-based MG [33], where the Fe-Fe pairs have strong magnetic interactions. More investigations are needed to settle this issue.

When going beyond the SRO, in contrast with the isostructural transition in elemental $\mathrm{Ce}$, we suggest that the change in MRO length scale behaves in a similar manner with the change in the SRO length scale [Figs. 5(c) and 5(d)]. Moreover, the change in MRO should be larger than the SRO, since the intercluster $\mathrm{Ce}-\mathrm{Ce}$ bonds (and or the linkage $\mathrm{Ce}$ atom in the liquidlike region) connecting nearest-neighbor SCCs have much lower binding strength than the atomic bonds within a SCC. Note that the MRO of MGs demonstrates the characteristics of a fractal network with a dimension of 
2.31 [4]. Zheng et al. [34] also found that the density- $Q_{1}$ relationship of MGs follows the fractional power law under high pressure. The present work uses temperature to modify the structure, instead of using composition and pressure as tuning variables in Refs. [4] and [34]. It would be interesting in the future to explore the similarity and difference of structure change induced by different physical parameters, such as pressure, temperature, and composition.

\section{CONCLUSION}

We present sufficient evidence to indicate an electrondelocalization transition-induced abnormal change of shortrange order, along with a unusual large-volume shrinkage in the Ce-based MGs. This is different from the isostructural transition in Ce metal, since the amorphous structure broadens the $4 f$ delocalization of $\mathrm{Ce}$ and diversifies the local environments around $\mathrm{Ce}$. Owing to the hierarchical bonding nature of the $\mathrm{Ce}-$ based MG, we suggest that the structure may change in a selfsimilar manner on different length scales in Ce-based MGs. The temperature-triggered local-structure change/transition is similar to the pressure-induced amorphous-amorphous transition suggested in the Ce-based MGs, in the sense that both process the same electronic delocalization mechanism [35-38]. Differently, the temperature-induced abnormal change occurs mainly on the topological and chemical shortrange orders $\left(Q_{2}\right.$ and $\left.Q_{3}\right)$, and under high pressure significant change of medium-range order $\left(Q_{1}\right)$ occurs. The present results shed light on the structure and the impact of electronic configuration of amorphous metals. Therefore, Ce-based and other rare-earth-based MGs provide a broad variety of systems, where the strongly correlated electronic behavior in amorphous systems and the interaction between $4 f$ configurations and amorphous structure under ambient/extreme conditions can be probed.

\section{ACKNOWLEDGMENTS}

The financial support from the National Natural Science Foundation of China (Grants No. 51371127 and No. 51274151), as well as additional support by the German Science Foundation under the Leibniz Program (Grant No. EC 111/26-1) and the ERC Advanced Grant INTELHYB (Grant No. ERC-2013-ADG-340025) is gratefully acknowledged. The authors would like to thank the European Synchrotron Radiation Facility (ESRF) in Grenoble, France, DESY (Hamburg, Germany), and the Beijing Synchrotron Radiation Facility (BSRF) in Beijing, China, for use of the synchrotron radiation facilities. Qiang Luo would like to thank the Alexander von Humboldt Foundation for financial support. The experimental assistance of Dr. Pieter Glatzel at ESRF and Dr. Liangliang Li at Center for High Pressure Science \& Technology Advanced Research China is greatly appreciated.

The authors declare no competing financial interests.
[1] W. H. Wang, C. Dong, and C. H. Shek, Bulk metallic glasses, Mater. Sci. Eng. R 44, 45 (2004).

[2] H. W. Sheng, W. K. Luo, F. M. Alamgir, J. M. Bai, and E. Ma, Atomic packing and short-to-medium range order in metallic glasses, Nature (London) 439, 419 (2006).

[3] D. B. Miracle, A structural model for metallic glasses, Nat. Mater. 3, 697 (2004).

[4] D. Ma, A. D. Stoica, and X. L. Wang, Power-law scaling and fractal nature of medium-range order in metallic glasses, Nat. Mater. 8, 30 (2009)

[5] J. Hwang, Nanoscale Structure and Structural Relaxation in $\mathrm{Zr}_{50} \mathrm{Cu}_{45} \mathrm{Al}_{5}$ Bulk Metallic Glass, Phys. Rev. Lett. 108, 195505 (2012).

[6] D. Ma, A. D. Stoica, X.-L. Wang, Z. P. Lu, B. Clausen, and D. W. Brown, Elastic Moduli Inheritance and the Weakest Link in Bulk Metallic Glasses, Phys. Rev. Lett. 108, 085501 (2012).

[7] K. Samwer, R. Busch, and W. L. Johnson, Change of Compressibility at the Glass Transition and Prigogine-Defay Ratio in ZrTiCuNiBe Alloys, Phys. Rev. Lett. 82, 580 (1999).

[8] T. Egami, S. J. Poon, Z. Zhang, and V. Keppens, Glass transition in metallic glasses: A microscopic model of topological fluctuations in the bonding network, Phys. Rev. B 76, 024203 (2007).

[9] A. R. Yavari, A. L. Moulec, A. Inoue, N. Nishiyama, N. Lupu, E. Matsubara, W. J. Botta, G. Vaughan, M. D. Michiel, and Å. Kvick, Excess free volume in metallic glasses measured by X-ray diffraction, Acta Mater. 53, 1611 (2005).

[10] S. Wei, F. Yang, J. Bednarcik, I. Kaban, O. Shuleshova, A. Meyer, and R. Busch, Liquid-liquid transition in a strong bulk metallic glass-forming liquid, Nat. Commun. 4, 3083 (2012).
[11] M. J. Duarte, P. Bruna, E. Pineda, D. Crespo, G. Garbarino, R. Verbeni, K. Zhao, W. H. Wang, A. H. Romero, and J. Serrano, Polyamorphic transitions in Ce-based metallic glasses by synchrotron radiation, Phys. Rev. B 84, 224116 (2011).

[12] H. W. Sheng, H. Z. Liu, Y. Q. Cheng, J. Wen, P. L. Lee, W. K. Luo, S. D. Shastrl, and E. Ma, Polyamorphism in a metallic glass, Nat. Mater. 6, 192 (2007).

[13] Q. S. Zeng, Y. Ding, W. L. Mao, W. Yang, S. V. Sinogeikin, J. Shu, H. Mao, and J. Z. Jiang, Origin of Pressure-Induced Polyamorphism in $\mathrm{Ce}_{75} \mathrm{Al}_{25}$ Metallic Glass, Phys. Rev. Lett. 104, 105702 (2010).

[14] S. R. Nagel and J. Tauc, Nearly-Free-Electron Approach to the Theory of Metallic Glass Alloys, Phys. Rev. Lett. 35, 380 (1975).

[15] G. Han, J. Qiang, F. Li, L. Yuan, S. Quan, Q. Wang, Y. Wang, C. Dong, and P. Haussler, The e/a values of ideal metallic glasses in relation to cluster formulae, Acta Mater. 59, 5917 (2011).

[16] C. C. Yuan, J. F. Xiang, X. K. Xi, and W. H. Wang, NMR Signature of Evolution of Ductile-To-Brittle Transition in Bulk Metallic Glasses, Phys. Rev. Lett. 107, 236403 (2011).

[17] X. K. Xi, M. T. Sandor, H. J. Wang, J. Q. Wang, W. H. Wang, and $\mathrm{Y}$. $\mathrm{Wu}$, Bonding characters of Al-containing bulk metallic glasses studied by ${ }^{27} \mathrm{Al}$ NMR, J. Phys.: Condens. Matter 23, 115501 (2011).

[18] P. Glatzel and U. Bergmann, High resolution 1s core hole X-ray spectroscopy in $3 \mathrm{~d}$ transition metal complexes-electronic and structural information, Coord. Chem. Rev. 249, 65 (2005).

[19] J.-P. Rueff, C. F. Hague, J.-M. Mariot, L. Journel, R. Delaunay, J.-P. Kappler, G. Schmerber, A. Derory, N. Jaouen, and G. Krill, $f$-State Occupancy at the $\gamma-\alpha$ Phase Transition of Ce-Th and Ce-Sc Alloys, Phys. Rev. Lett. 93, 067402 (2004). 
[20] M. B. Tang, J. Q. Wang, W. H. Wang, L. Xia, K. C. Chan, and J. T. Zhao, Valence fluctuation and electron-phonon coupling in $\mathrm{La}_{68-\mathrm{x}} \mathrm{Ce}_{\mathrm{x}} \mathrm{Al}_{10} \mathrm{Cu}_{20} \mathrm{Co}_{2}(\mathrm{x}=0,34$, and 68) metallic glasses, J. Appl. Phys. 108, 033525 (2010).

[21] P. Yu, R. J. Wang, D. Q. Zhao, and H. Y. Bai, Anomalous temperature dependent elastic moduli of Ce-based bulk metallic glass at low temperatures, Appl. Phys. Lett. 91, 201911 (2007).

[22] P. Yu, R. J. Wang, D. Q. Zhao, and H. Y. Bai, Temperature dependence of elastic moduli in bulk metallic glasses down to liquid nitrogen temperature, Appl. Phys. Lett. 90, 251904 (2007).

[23] N. Devaux, M. Casula, F. Decremps, and S. Sorella, Electronic origin of the volume collapse in cerium, Phys. Rev. B 91, 081101 (2015).

[24] J. W. Allen and R. M. Martin, Kondo Volume Collapse and the $\gamma \rightarrow \alpha$ Transition in Cerium, Phys. Rev. Lett. 49, 1106 (1982).

[25] M. Lavagna, C. Lacroix, and M. Cyrot, Volume collapse in the Kondo lattice, Phys. Lett. A 90, 210 (1982).

[26] F. Decremps, L. Belhadi, D. L. Farber, K. T. Moore, F. Occelli, M. Gauthier, A. Polian, D. Antonangeli, C. M. Aracne-Ruddle, and B. Amadon, Diffusionless $\gamma \rightleftarrows \alpha$ Phase Transition in Polycrystalline and Single-Crystal Cerium, Phys. Rev. Lett. 106, 065701 (2011).

[27] M. Casadei, X. Ren, P. Rinke, A. Rubio, and M. Scheffler, Density-Functional Theory for f-Electron Systems: The $\alpha-\gamma$ Phase Transition in Cerium, Phys. Rev. Lett. 109, 146402 (2012).

[28] J. Bieder and B. Amadon, Thermodynamics of the $\alpha-\gamma$ transition in cerium from first principles, Phys. Rev. B 89, 195132 (2014).

[29] T. Egami, Atomic level stresses, Prog. Mater. 56, 637 (2011).

[30] P. Guan, M. Chen, and T. Egami, Stress-Temperature Scaling for Steady-State Flow in Metallic Glasses, Phys. Rev. Lett. 104, 205701 (2010).
[31] O. O. Bernal, D. E. MacLaughlin, H. G. Lukefahr, and B. Andraka, Copper NMR and Thermodynamics of $\mathrm{UCu}_{5-\mathrm{x}} \mathrm{Pd}_{\mathrm{x}}$ : Evidence for Kondo Disorder, Phys. Rev. Lett. 75, 2023 (1995).

[32] M. B. Tang, H. Y. Bai, W. H. Wang, D. Bogdanov, K. Winzer, K. Samwer, and T. Egami, Heavy-fermion behavior in ceriumbased metallic glasses, Phys. Rev. B 75, 172201 (2007).

[33] F. E. Luborsky and J. L.Walter, Magnetically induced anisotropy in amorphous alloys of Fe-Ni-P-B, IEEE Trans. Magn. 13, 1635 (1977).

[34] Q. S. Zheng, Y. Kono, Y. Lin, Z. Zeng, J. Wang, S. V. Sinogeikin, C. Park, Y. Meng, W. Yang, H.-K. Mao, and W. L. Mao, Universal Fractional Noncubic Power Law for Density of Metallic Glasses, Phys. Rev. Lett. 112, 185502 (2014).

[35] L. Belhadi, F. Decremps, S. Pascarelli, L. Cormier, Y. Le Godec, S. Gorsse, F. Baudelet, C. Marini, and G. Garbarino, Polyamorphism in cerium based bulk metallic glasses: Electronic and structural properties under pressure and temperature by $\mathrm{x}$-ray absorption techniques, Appl. Phys. Lett. 103, 111905 (2013).

[36] G. Li, Y. Y. Wang, P. K. Liaw, Y. C. Li, and R. P. Liu, Electronic Structure Inheritance and Pressure-Induced Polyamorphism in Lanthanide-Based Metallic Glasses, Phys. Rev. Lett. 109, 125501 (2012).

[37] Q. Luo, G. Garbarino, B. Sun, D. Fan, Y. Zhang, Z. Wang, Y. Sun, J. Jiao, X. Li, P. Li, N. Mattern, J. Eckert, and J. Shen, Hierarchical densification and negative thermal expansion in Cebased metallic glass under high pressure, Nat. Commun. 6, 5703 (2015).

[38] F. Decremps, G. Morard, G. Garbarino, and M. Casula, Polyamorphism of a Ce-based bulk metallic glass by highpressure and high-temperature density measurements, Phys. Rev. B 93, 054209 (2016). 